\title{
Muscle Cramp, CTCAE
}

National Cancer Institute

\section{Source}

National Cancer Institute. Muscle Cramp, CT CAE. NCI Thesaurus. Code C143224.

A disorder characterized by marked cramping sensation originating from a muscle or group of muscles. 